\title{
原位聚合三维陶瓷骨架增强全固态锂电池电解质
}

\author{
颜一垣 ${ }^{1}$, 鞠江伟 ${ }^{2}$, 于美燕 ${ }^{1}$, 陈守刚 ${ }^{1}$, 崔光䂞 ${ }^{2}$ \\ (1. 中国海洋大学 材料科学与工程学院, 青岛 266100; 2. 中国科学院 青岛生物能源与过程研究所, 青岛 \\ 266101)
}

摘 要: 有机/无机复合电解质被认为是全固态锂电池中最具潜力的固态电解质之一, 但由于无机填料易团聚, 通过提 高无机填料含量来改善复合电解质的电导率难有成效。此外, 在全固态锂电池中, 电解质和电极之间松散的固-固接 触造成过大的界面阻抗，限制了全固态锂电池的性能。本研究采用固相法合成具有 $\mathrm{Li}^{+}$连续传输通道的自支撑三维多 孔 $\mathrm{Li}_{6.4} \mathrm{Al}_{0.1} \mathrm{La}_{3} \mathrm{Zr}_{1.7} \mathrm{Ta}_{0.3} \mathrm{O}_{12}$ 骨架, 并利用原位聚合的方法构筑一体化电解质/电极固-固界面。此策略指导合成的复合电 解质的室温电导率可达 $1.9 \times 10^{-4} \mathrm{~S} \cdot \mathrm{cm}^{-1}$ 。同时，一体化的界面使得 $\mathrm{Li}-\mathrm{Li}$ 对称电池的界面阻抗从 $1540 \Omega \cdot \mathrm{cm}^{2}$ 降低至 449 $\Omega \cdot \mathrm{cm}^{2}$, 因此 $4.3 \mathrm{~V}\left(v s . \mathrm{Li}^{+} / \mathrm{Li}\right)$ 的 $\mathrm{LiCoO}_{2} \mid \mathrm{Li}$ 全固态锂电池展现出良好的电化学性能。

关 键 词: 固态复合电解质; 原位聚合; 多孔骨架; 全固态电池

中图分类号: TQ174 文献标识码: A

\section{In-situ Polymerization Integrating 3D Ceramic Framework in All Solid-state Lithium Battery}

\author{
YAN Yiyuan $^{1}$, JU Jiangwei ${ }^{2}$, YU Meiyan ${ }^{1}$, CHEN Shougang ${ }^{1}$, CUI Guanglei ${ }^{2}$
}

(1. School of Materials Science and Engineering, Ocean University of China, Qingdao 266100, China; 2. Qingdao Institute of Bioenergy and Bioprocess Technology, Chinese Academy of Sciences, Qingdao 266101, China)

\begin{abstract}
Organic/inorganic composites have been considered as promising electrolyte candidates in all solid-state lithium batteries. Aiming at improving the conductivity significantly by increasing the frequently-used $0 \mathrm{D}$ or $1 \mathrm{D}$ ceramic nano-fillers to high content is unsuccessful due to the particle tendency to agglomeration. What's worse, the loose contact between the solid electrolyte and solid electrodes is much of a serious barrier to the performance and thus to the application of all solid-state lithium batteries. Herein, self-supported $3 \mathrm{D}$ porous $\mathrm{Li}_{6.4} \mathrm{Al}_{0.1} \mathrm{La}_{3} \mathrm{Zr}_{1.7} \mathrm{Ta}_{0.3} \mathrm{O}_{12}$ frameworks are employed to provide percolated fast $\mathrm{Li}^{+}$conductive pathway while in-situ polymerization of poly(ethylene glycol) methyl ether acrylate can integrate the loose solid-solid interface and reduce the interfacial resistance efficiently. Inspiringly, the $\mathrm{Li}^{+}$conductivity of the composite exhibits $1.9 \times 10^{-4} \mathrm{~S} \cdot \mathrm{cm}^{-1}$ at room temperature. The interfacial resistance in Li-Li batteries decreases significantly from 1540 to $449 \Omega \cdot \mathrm{cm}^{2}$, rendering good capacity and cyclability of the $4.3 \mathrm{~V}$ (vs. $\mathrm{Li}^{+} / \mathrm{Li}$ ) $\mathrm{LiCoO}_{2} \mid \mathrm{Li}$ all solid-state lithium battery.
\end{abstract}

Key words: solid composite electrolyte; in-situ polymerization; porous framework; all solid-state battery

作为新一代储能器件, 锂离子电池在手机、电 动车、电网等领域备受青睐。然而，传统锂离子电

收稿日期: 2020-03-23; 收到修改稿日期：2020-05-11

基金项目: 国家自然科学基金(51902325) National Natural Science Foundation of China(51902325)

作者简介：颜一垣(1994-), 男, 硕士研究生. E-mail: yanyiyuan94@163.com YAN Yiyuan(1994-), male, Master candidate. E-mail: yanyiyuan94@163.com

通讯作者: 陈守刚, 教授. E-mail: sgchen@ouc.edu.cn; 崔光否, 研究员. E-mail: cuigl@qibebt.ac.cn CHEN Shougang, professor. E-mail: sgchen@ouc.edu.cn; CUI Guanglei, professor. E-mail: cuigl@qibebt.ac.cn 
池所使用的有机电解液存在泄露、易燃易爆等安全 隐患 ${ }^{[1-3]}$ 。解决该安全性问题最有效的方法是将电解 液固态化, 得到高安全性的全固态锂电池 (All solid-state lithium battery, ASLB)。此外, 固态电解质 具有较高的机械强度, 使利用锂金属(Li)作为负极 成为可能, 从而大幅度提高 ASLB 的能量密度 ${ }^{[4-5]}$ 。 目前, 主要研究的固态电解质有两大类: 聚合物电 解质 (Solid polymer electrolyte, SPE)和无机固态电 解质(Solid inorganic electrolyte, SIE $)^{[6]}$ 。以聚环氧乙 烯(poly(ethylene oxide), PEO)为代表的 SPE 柔韧性 好, 且能与电极相容。但低的室温离子电导率 $\left(\sigma_{\mathrm{Li}^{+}}\right.$, $\left.10^{-6} \sim 10^{-8} \mathrm{~S} \cdot \mathrm{cm}^{-1}\right)$ 限制了其应用 ${ }^{[7]}$ 。相反, 以氧化物, 如 $\mathrm{Li}_{7} \mathrm{La}_{3} \mathrm{Zr}_{2} \mathrm{O}_{12}$ (LLZO)、 $\mathrm{Li}_{1.3} \mathrm{Al}_{0.3} \mathrm{Ti}_{1.7}\left(\mathrm{PO}_{4}\right)_{3}$ (LATP) 为代表的 SIE 具有较高的 $\sigma_{\mathrm{Li}^{+}}\left(10^{-4} \sim 10^{-2} \mathrm{~S} \cdot \mathrm{cm}^{-1}\right)^{[8-11]}$ 。 然而, SIE 硬而脆的特性导致 ASLB 组装困难, 同样 限制了其在 ASLB 中的应用 ${ }^{[1,12]}$ 。因此, 单一使用 SPE 或 SIE 都无法获得高性能 ASLB。

为弥补单一固态电解质的缺陷, 结合二者的优 势, 将 SIE 与 SPE 进行复合是一种行之有效的方法。 SPE 中引入零维 SIE 颗粒或者一维 SIE 纤维能够降 低 SPE 基体的结晶度或玻璃化转变温度 ${ }^{[13-14]}$, 一般 可将 SPE 的电导率提升一个数量级 ${ }^{[15]}$ 。但是零维或 一维 SIE 填料的含量过高会产生团聚, 导致电导率 降低 ${ }^{[16-17]}$ 。并且, 高电导率的 SIE 填料或是被 SPE 相孤立或是被有机/无机界面相孤立, 阻碍了 $\mathrm{Li}^{+}$在 高电导率 SIE 相中的快速传导。不同于将 SIE 填料 分散于 SPE 中, 相反, 将 SPE 浇注于多孔 SIE 骨架 中, 即三维 SIE 填料中, 可得到具有连续 SIE 相的有 机/无机复合电解质 ${ }^{[17-18]}$ 。这种结构不仅能有效避免 SIE 填料的团聚, 还能为 $\mathrm{Li}^{+}$的快速传输提供连续通 道, 大幅提升电导率 ${ }^{[19]}$ 。

虽然三维 SIE 骨架可以大幅提高复合电解质的 $\sigma_{\mathrm{Li}^{+}}$, 但 ASLB 的性能仍受限于固态电解质与电极 之间的间隙(图 1(a))所导致的高界面阻抗 ${ }^{[20]}$ 。因此, 构筑一体化固-固界面与提升 $\sigma_{\mathrm{Li}^{+}}$对于高性能

ASLB 都具有重要的意义。前期工作已证明原位聚 合可以成功实现一体化电极/电解质界面的构筑, 并 显著降低 ASLB 的界面阻抗 ${ }^{[21]}$ 。原位聚合过程中, 液态单体能够将三维骨架完全填充, 在任意尺度上 与电池内部的各部分产生良好的界面接触。随后, 单体聚合为 SPE, 并保留液-固界面紧密接触的优 势, 形成一体化的固-固界面(图 1(b)) ${ }^{[22]}$ 。基于此, 本工作利用高电导率 $\mathrm{Li}_{6.4} \mathrm{Al}_{0.1} \mathrm{La}_{3} \mathrm{Zr}_{1.7} \mathrm{Ta}_{0.3} \mathrm{O}_{12}$ (LLZTO) 制备三维多孔骨架(p-LLZTO); 利用与 Li 相容性良 好的聚乙二醇甲基醚丙烯酸酯(poly(ethylene glycol)
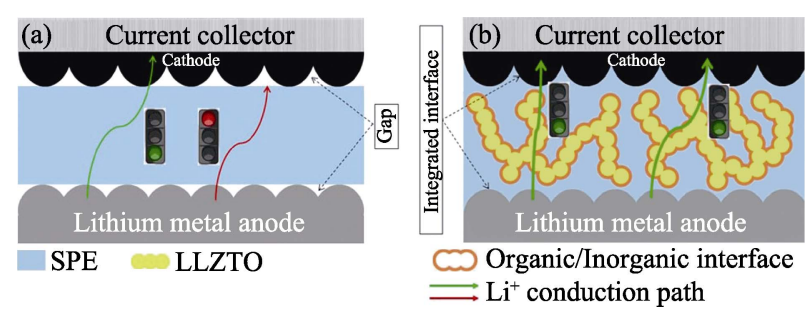

图 1 (a)非原位聚合策略和(b)原位聚合策略制备的 ASLB 内部结构示意图

Fig. 1 Schematic illustration of ASLB structure prepared via (a) ex-situ and (b) in-situ methods with p-LLZTO as ceramic fillers

methyl ether acrylate, PEGMEA, $\mathrm{H}_{3} \mathrm{C} \mathrm{O}_{\mathrm{n}} \mathrm{f}_{\mathrm{n}} \mathrm{O}=\mathrm{CH}_{2}$, $n=8 \sim 9$ )作为原位聚合的单体 ${ }^{[23-24]}$ 。如图 1(b)所示, p-LLZTO 能够提供连续快速的 $\mathrm{Li}^{+}$传输通道, 而聚 合后的 PEGMEA, 即 P(PEGMEA), 能够在原本接 触差的电极和 P(PEGMEA)/p-LLZTO 复合电解质 (3D composite)之间构筑一体化界面, 从而获得以钴 酸锂 $\left(\mathrm{LiCoO}_{2}\right)$ 为正极, $\mathrm{Li}$ 为负极的 $\mathrm{ASLB}$ 。

\section{1 实验方法}

固相反应法合成 LLZTO 粉末: 将 $\mathrm{Al}_{2} \mathrm{O}_{3} 、 \mathrm{ZrO}_{2}$ 、 $\mathrm{LiOH} 、 \mathrm{Ta}_{2} \mathrm{O}_{5}$ 及 $\mathrm{La}_{2} \mathrm{O}_{3}$ 按照 $\mathrm{Li}_{6.4} \mathrm{Al}_{0.1} \mathrm{La}_{3} \mathrm{Zr}_{1.7} \mathrm{Ta}_{0.3} \mathrm{O}_{12}$ 的化学计量比称量后倒入球磨罐中。以异丙醇为球 磨介质, 并于 $350 \mathrm{r} / \mathrm{min}$ 的速率球磨 $10 \mathrm{~h}$ 。之后, 将 球磨所得浆料置于 $60{ }^{\circ} \mathrm{C}$ 烘箱中干燥, 并于 $1000{ }^{\circ} \mathrm{C}$ 的空气氛围中烧结 $5 \mathrm{~h}$, 再将烧结所得块体进行研 磨即可得到 LLZTO 粉末。

将上述所得 LLZTO 粉末与造孔剂石墨粉以质 量比 $3.5: 1.5$ 均匀混合后, 在 $12 \mathrm{MPa}$ 的压力下压制 成片。再将其置于氧化镁瓷舟中, 并用 LLZTO 粉末 包覆, 在 $1150{ }^{\circ} \mathrm{C}$ 的空气氛围中烧结 $5 \mathrm{~h}$, 得到 p-LLZTO。直接将上述所得 LLZTO 粉末于 $12 \mathrm{MPa}$ 压力下压成片, 置于氧化美瓷舟中, 并用 LLZTO 粉 末包裹, 于 $1150{ }^{\circ} \mathrm{C}$ 的空气氛围中烧结 $5 \mathrm{~h}$, 可得致 密 LLZTO 样品。

为证明原位聚合对 ASLB 的积极作用, 本工作 对原位聚合及非原位聚合的 $\mathrm{LiCoO}_{2} \mid \mathrm{Li}$ ASLB 性能 进行对比。原位聚合 $\mathrm{LiCoO}_{2} \mid \mathrm{Li}$ ASLB 的制备: 将 p-LLZTO 或纤维素隔膜置于锂片上, 滴加 $100 \mu \mathrm{L}$ 的 PEGMEA 前驱体溶液, 放置正极片, 在手套箱中完 成电池的组装后, 移入 $60{ }^{\circ} \mathrm{C}$ 烘箱中, 加热完成原 位聚合。非原位聚合 $\mathrm{LiCoO}_{2} \mid \mathrm{Li} \mathrm{ASLB}$ 的制备: 先将 p-LLZTO 置于聚四氟乙烯板上, 然后滴加 $100 \mu \mathrm{L}$ PEGMEA 前驱体溶液, 在 $60{ }^{\circ} \mathrm{C}$ 加热使 PEGMEA 完 成聚合。将 $3 \mathrm{D}$ composite 从聚四氟乙烯板上取下并 
置于锂片和正极片之间，完成非原位电池组装。

\section{2 结果与讨论}

\section{$2.1 p$-LLZTO 的表征}

本工作中所制备的 p-LLZTO 和 LLZTO 粉末的 $X$ 射线衍射(X-ray diffraction, XRD) 图谱(图 2(a))一 致, 并与标准石榴石型的衍射峰吻合, 说明本工作 成功合成了 LLZTO, 且石墨粉在烧结过程中不与 LLZTO 发生反应, 无残留。扫描电子显微镜 (Scanning electron microscope, SEM)照片(图 2(b))清 晰地显示出 p-LLZTO 的多孔结构。利用压录法对 p-LLZTO 的微结构特征进一步分析 (图 2(c)), p-LLZTO 的孔径分布范围为 $0.5 \sim 10 \mu \mathrm{m}$, 平均孔径 为 $4.14 \mu \mathrm{m}$, 孔隙率和曲折因子分别为 $58 \%$ 和 2.14 。 根据室温下电化学阻抗图谱(Electrochemical impedance spectroscopy, EIS)(图 2(d))的结果计算可得, 致密 LLZTO 的室温 $\sigma_{\mathrm{Li}^{+}}$为 $2.4 \times 10^{-4} \mathrm{~S} \cdot \mathrm{cm}^{-1}$, $\mathrm{p}$-LLZTO 的室温 $\sigma_{\mathrm{Li}^{+}}$为 $4.3 \times 10^{-5} \mathrm{~S} \cdot \mathrm{cm}^{-1}$ 。具有高孔 隙率和低曲折因子的 p-LLZTO 能够保证液态单体 在电池中顺利渗透, 并在原位聚合后形成一体化界 面。同时, $\mathrm{p}$-LLZTO 能够为 $\mathrm{Li}^{+}$提供快速的传输通道,
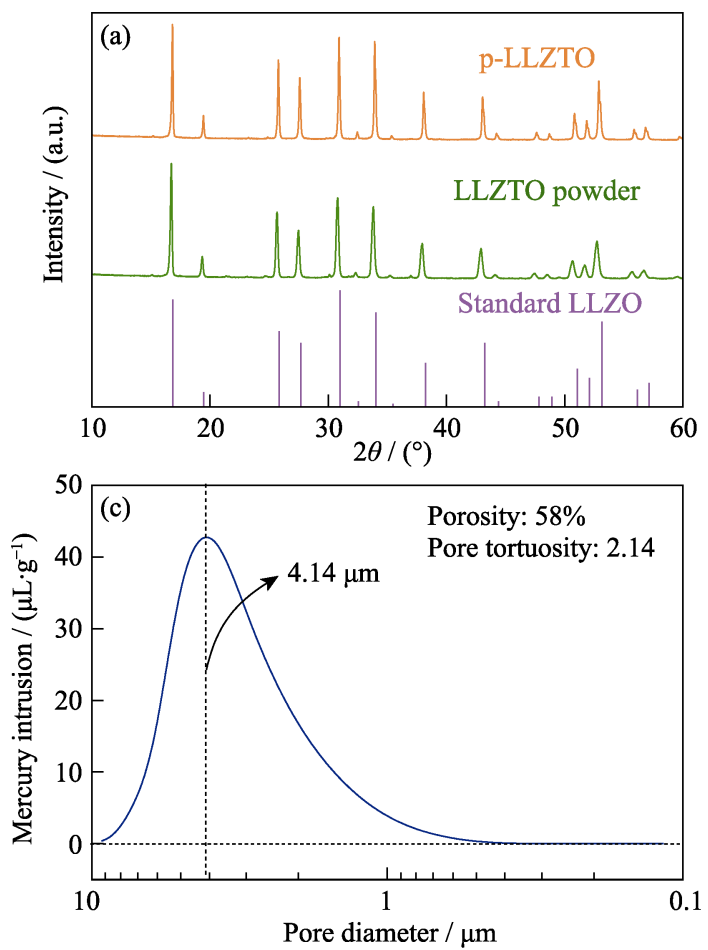

大幅提高 $\mathrm{P}(\mathrm{PEGMEA})$ 的 $\sigma_{\mathrm{Li}^{+}}$, 为制备高性能的 ASLB 提供保障。

\section{$2.23 D$ composite 的表征}

首先使用傅里叶红外光谱(Fourier transform infrared spectrometer, FT-IR)分析 PEGMEA 的聚合程 度。在 $60{ }^{\circ} \mathrm{C}$ 加热 $24 \mathrm{~h}$ 后, 如图 3(a)所示, 位于 $1620 \mathrm{~cm}^{-1}$ 附近的 $\mathrm{C}=\mathrm{C}$ 峰完全消失, 而其它官能团, 如 $\mathrm{C}=\mathrm{O}$ 或者 $\mathrm{C}-\mathrm{O}-\mathrm{C}$ 的峰则仍保留，证明在该条件 下 PEGMEA 能够完全聚合, 转化为 P(PEGMEA)。 而 3D composite 中的 P(PEGMEA) 与纯 P(PEGMEA) 的红外光谱几乎相同, 说明 LLZTO 不影响 PEGMEA 的聚合且不与 PEGMEA 反应。为进一步 分析 P(PEGMEA)的分子结构, 又对 PEGMEA 及 P(PEGMEA)进行核磁共振测试(Nuclear magnetic resonance, NMR)测试。在 PEGMEA 的氢谱(图 3(b)) 中, 与 $\mathrm{C}=\mathrm{C}$ 相连氢原子的峰分别位于 $6.4 、 6.2$ 和 6.0 。 在 $60{ }^{\circ} \mathrm{C}$ 加热 $24 \mathrm{~h}$ 后, 这些峰完全消失, 同时在 2.5 1.5 的位置上出现了若干峰。这归结于 $\mathrm{C}=\mathrm{C}$ 双 键被打开，同样证明 PEGMEA 完成了聚合。

除 FT-IR 和 NMR 外, 另一个能证明聚合完成的 有力证据是电池阻抗随加热时间的变化关系。图 3(c) 为在 $60{ }^{\circ} \mathrm{C}$ 下 steel $\mid 3 \mathrm{D}$ composite|steel 欧姆阻抗与加
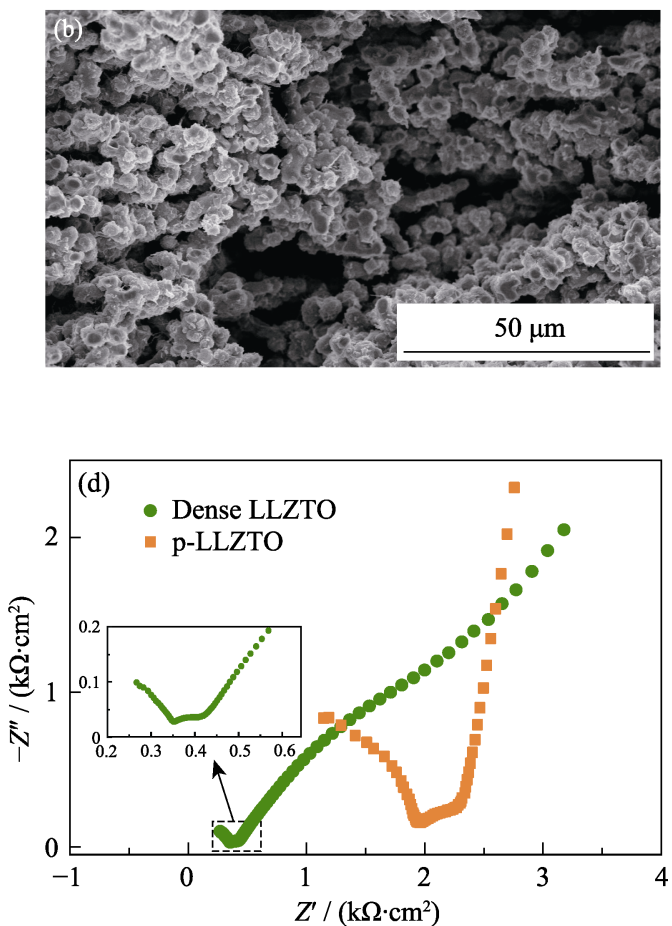

图 2 (a)标准 LLZO 及本实验制备的 LLZTO 粉末和 p-LLZTO 的 XRD 图谱; (b) p-LLZTO 的截面 SEM 照片; (c)p-LLZTO 的 孔径分布曲线; (d)致密 LLZTO 和 p-LLZTO 的室温阻抗图谱(插图: 局部放大的致密 LLZTO 阻抗谱)

Fig. 2 (a) XRD patterns of standard LLZO, the as-prepared LLZTO powders and p-LLZTO; (b) Cross sectional SEM image of p-LLZTO; (c) Pore size distribution of p-LLZTO; (d) EIS plots of dense LLZTO and p-LLZTO at room temperature with inset showing the partial magnified spectrum of the dense LLZTO 

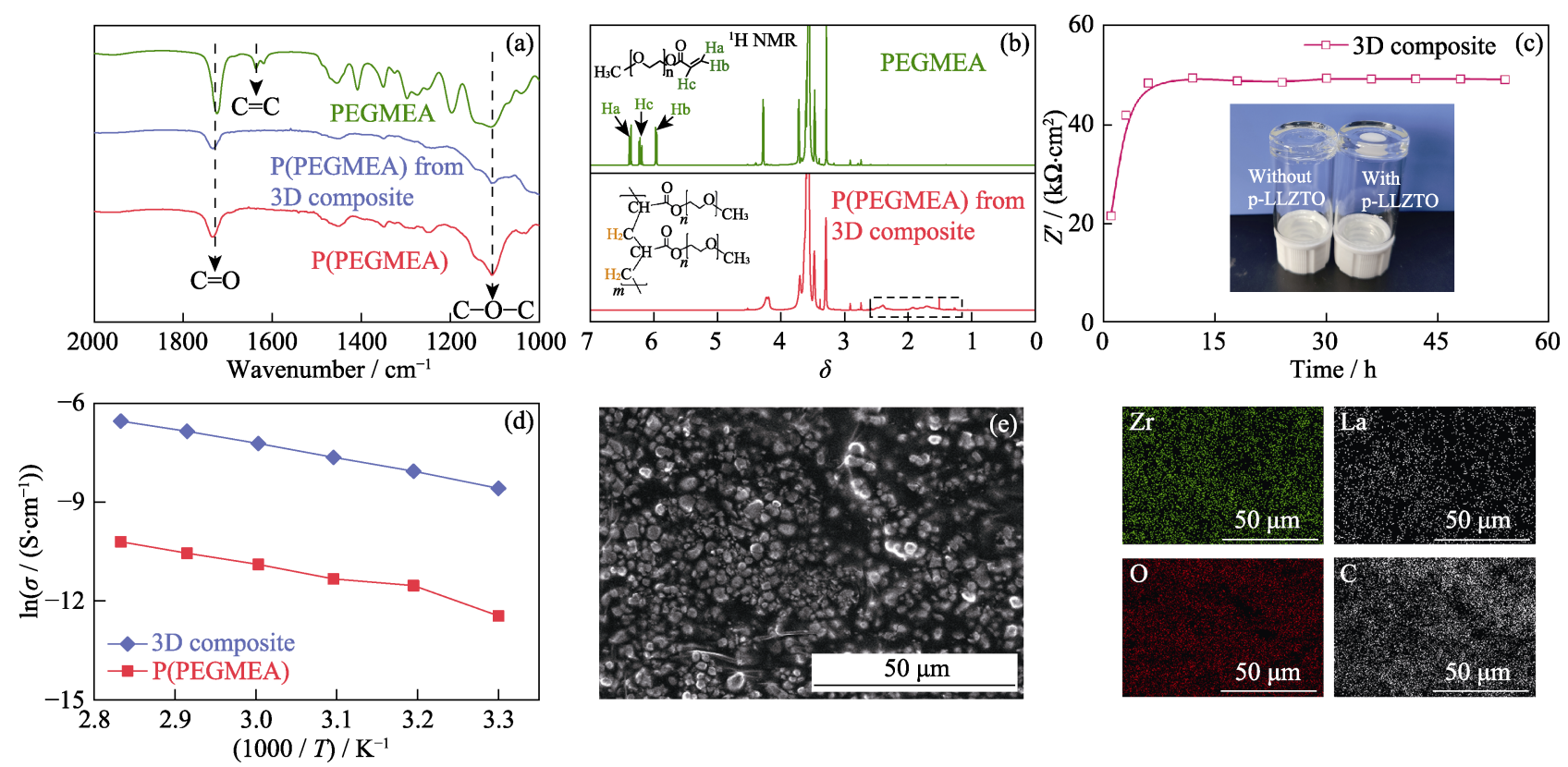

图 3 (a)PEGMEA、P(PEGMEA)和 3D composite 中 P(PEGMEA)的红外图谱; (b)PEGMEA 和 3D composite 中 P(PEGMEA)的 核磁共振氢谱及相关结构式(溶剂为氛代 $N, N$-二甲基甲酰胺); (c)60 ${ }^{\circ} \mathrm{C}$ 条件下 steel|3D composite|steel 电池欧姆阻抗与加热时 间关系曲线，插图为有/无 p-LLZTOP 的 PEGMEA 在小瓶中 $60{ }^{\circ} \mathrm{C}$ 加热 $24 \mathrm{~h}$ 后的照片; (d)P(PEGMEA)和 3D composite 的电导 率与温度的关系曲线; (e)3D composite 的截面 SEM 照片及元素分布图

Fig. 3 (a) FT-IR spectra of PEGMEA, P(PEGMEA), and P(PEGMEA) from the 3D composite; (b) ${ }^{1} \mathrm{H}$ NMR spectra of PEGMEA and $\mathrm{P}$ (PEGMEA) from the $3 \mathrm{D}$ composite(the solvents are deuterated $N, N$-dimethylformamide) with insets showing the corresponding structural formula of PEGMEA and P(PEGMEA); (c) Thermal evolution of ohmic resistance at $60{ }^{\circ} \mathrm{C}$ for steel|3D composite|steel symmetrical cell with inset showing the digital image of PEGMEA with/without p-LLZTO after heat-treatment at

$60{ }^{\circ} \mathrm{C}$ for $24 \mathrm{~h}$; (d) Relation between ionic conductivity of electrolyte and temperature for P(PEGMEA) and 3D composite; (e) Cross sectional SEM image and element mapping analysis of the $3 \mathrm{D}$ composite

表 1 不同固态电解质的室温电导率 $\left(\sigma_{\mathrm{Li}^{+}}\right)$

Table 1 Conductivities $\left(\sigma_{\mathrm{Li}^{+}}\right)$of different solid electrolytes at room temperature

\begin{tabular}{ccccccc}
\hline Electrolyte & Lithium salt & $\mathrm{EO}^{\mathrm{a}}: \mathrm{Li}^{+}$ & $\begin{array}{c}\text { Conductivity of } \\
\text { polymer } /\left(\mathrm{S} \cdot \mathrm{cm}^{-1}\right)\end{array}$ & $\begin{array}{c}\text { Conductivity of } \\
\text { composite } /\left(\mathrm{S} \cdot \mathrm{cm}^{-1}\right)\end{array}$ & Promotion factor & Ref. \\
\hline PEO/LATP particles & LiClO $_{4}$ & $15: 1$ & $1.3 \times 10^{-6}$ & $9.5 \times 10^{-6}$ & 7.5 & {$[25]$} \\
PEO/LLZO fibers & LiTFSI $^{\mathrm{b}}$ & - & $2.5 \times 10^{-6}$ & $2.7 \times 10^{-5}$ & 11 & {$[26]$} \\
PEO/LATP fibers & LiTFSI & $8: 1$ & $3.2 \times 10^{-6}$ & $4.9 \times 10^{-5}$ & 15 & {$[27]$} \\
PEO/3D LLZO & LiTFSI & $10: 1$ & $1.8 \times 10^{-6}$ & $8.5 \times 10^{-5}$ & 47 & {$[19]$} \\
PEO/3D LLTO & LiTFSI & $10: 1$ & $2.2 \times 10^{-6}$ & $8.8 \times 10^{-5}$ & 40 & {$[18]$} \\
\hline
\end{tabular}

a: ethylene oxide(- $\left.\mathrm{CH}_{2}-\mathrm{CH}_{2}-\mathrm{O}-\right)$; b: lithium bis(trifluoromethanesulfonyl)imide); c: $\mathrm{Li}_{1.4} \mathrm{Al}_{0.4} \mathrm{Ti}_{1.6}\left(\mathrm{PO}_{4}\right)_{3} ; \mathrm{d} \mathrm{Li}_{0.35} \mathrm{La}_{0.55} \mathrm{TiO}_{3}$

热时间的关系曲线。PEGMEA 在加热过程中聚合固 化阻碍 $\mathrm{Li}^{+}$传输, 其电池的欧姆阻抗在最初的 $12 \mathrm{~h}$ 内迅速从 $21 \Omega \cdot \mathrm{cm}^{2}$ 增加至 $49 \Omega \cdot \mathrm{cm}^{2}$ 。12 h 后, 其欧 姆阻抗基本保持不变, 说明 PEGMEA 已经完全聚 合。根据 EIS 结果, 3D composite 在室温和 $60{ }^{\circ} \mathrm{C}$ 的 电导率分别为 $1.9 \times 10^{-4}$ 和 $7.3 \times 10^{-4} \mathrm{~S} \cdot \mathrm{cm}^{-1}$ (图 3(d)), 为 $\mathrm{P}(\mathrm{PEGMEA})$ 室温电导率 $\left(3.6 \times 10^{-6} \mathrm{~S} \cdot \mathrm{cm}^{-1}\right)$ 的 53 倍。该结果与文献 ${ }^{[18-19,25-27]}$ 报道相符(表 1)。SPE 基 体中引入 SIE 颗粒时, 其电导率提升因子(定义为复 合电解质的电导率与 SPE 电导率之比)为 7.5 11。
而当 SIE 填料为三维骨架时, 其 $\sigma_{\mathrm{Li}^{+}}$能够从 $1.8 \times 10^{-6} \mathrm{~S} \cdot \mathrm{cm}^{-1}$ 提升至 $8.5 \times 10^{-5} \mathrm{~S} \cdot \mathrm{cm}^{-1}$, 电导率提 升因子高达 47 。电导率的大幅提升一方面是由于高 电导率的无机相能够为 $\mathrm{Li}^{+}$的传输提供连续通道, 即 连续的 LLZTO 及 LLZTO/P(PEGMEA) 界面 ${ }^{[17-19,28]}$ 。 另一方面则是得益于 $3 \mathrm{D}$ composite 致密的结构。如 图 3(e)所示, La、 $\mathrm{Zr} 、 \mathrm{C}$ 和 $\mathrm{O}$ 的元素分布证明 SIE 填料在 3D composite 中分布均匀, 并被 P(PEGMEA) 完全包裹, 无孔隙。此外, 图 3(c)中 P(PEGMEA)在 $60{ }^{\circ} \mathrm{C}$ 加热 $24 \mathrm{~h}$ 后固化的照片也能够说明 PEGMEA 
聚合完全。基于以上分析, 实验中相关电池首先在 $60{ }^{\circ} \mathrm{C}$ 下加热 $24 \mathrm{~h}$ 以确保 PEGMEA 完全聚合。

电化学窗口和锂离子迁移数 $\left(t_{\mathrm{Li}^{+}}\right)$也是电解质 的重要性质。图 S1 中显示 $3 \mathrm{D}$ composite 的分解电 压高于 $4.5 \mathrm{~V}\left(v s . \mathrm{Li}^{+} / \mathrm{Li}\right)$, 表明 3D composite 能够用 于高电压的正极体系。而高 $t_{\mathrm{Li}^{+}}$对于降低极化和抑 制锂枝晶生长具有重要意义。通过恒流极化的方法 (图 S2) ${ }^{[21]}$, 得到 $3 \mathrm{D}$ composite 的 $t_{\mathrm{Li}^{+}}$为 0.43 , 约为 $\mathrm{P}(\mathrm{PEGMEA})\left(t_{\mathrm{Li}^{+}}=0.12\right)$ 的 3.5 倍。高的 $t_{\mathrm{Li}^{+}}$归因于 $3 \mathrm{D}$ composite 中 $42 \mathrm{vol} \%$ 的 $\operatorname{LLZTO}\left(t_{\mathrm{Li}^{+}}=1\right)$ 。高室温 $\sigma_{\mathrm{Li}^{+}}$和宽电压窗口和高 $t_{\mathrm{Li}^{+}}$表明 $3 \mathrm{D}$ composite 在 ASLB 中的应用极具潜力。

\section{$2.33 D$ composite 与 $\mathrm{Li}$ 的相容性}

对 $3 \mathrm{D}$ composite 与 $\mathrm{Li}$ 的相容性进行测试以确定
其能否应用于 $\mathrm{ASLB}^{[12]}$ 。本实验对 $\mathrm{Li} \mid 3 \mathrm{D}$ composite $\mid \mathrm{Li}$ 进行 EIS 和恒流极化测试, 并以 Li|P(PEGMEA)|Li 和 $\mathrm{Li}|\mathrm{LLZTO}| \mathrm{Li}$ 作为对照。图 4(a f) 为基于不同电解 质的电池在热处理前后的 EIS 图谱。加热前 Li|PEGMEA|Li 的 EIS 图谱由一个半圆和一条斜线 组成，半圆与实轴的交点为欧姆阻抗，而半圆的跨 度为界面阻抗 ${ }^{[29-30]}$ 。 Li 3 D composite $\mid \mathrm{Li}$ 的 EIS 图谱 中存在两个半圆, 这可能是固态 p-LLZTO 所致。在 $60{ }^{\circ} \mathrm{C}$ 加热 $24 \mathrm{~h}$ 后, Li|P(PEGMEA)|Li 电池的 EIS 图 谱同样出现两个半圆, 高频区半圆的跨度为欧姆阻 抗，而中频区半圆的跨度为界面阻抗 ${ }^{[31]}$ 。

根据阻抗图谱的拟合结果，上述电池热处理前 后欧姆阻抗及界面阻抗的变化总结于图 4(g,h)中。加 热前, $\mathrm{Li} \mid 3 \mathrm{D}$ composite $\mid \mathrm{Li}$ 的欧姆阻抗为 $2736 \Omega \cdot \mathrm{cm}$, 仅 为 $\mathrm{Li}|\mathrm{PEGMEA}| \mathrm{Li}$ 欧姆阻抗 $8550 \Omega \cdot \mathrm{cm}$ 的三分之一。
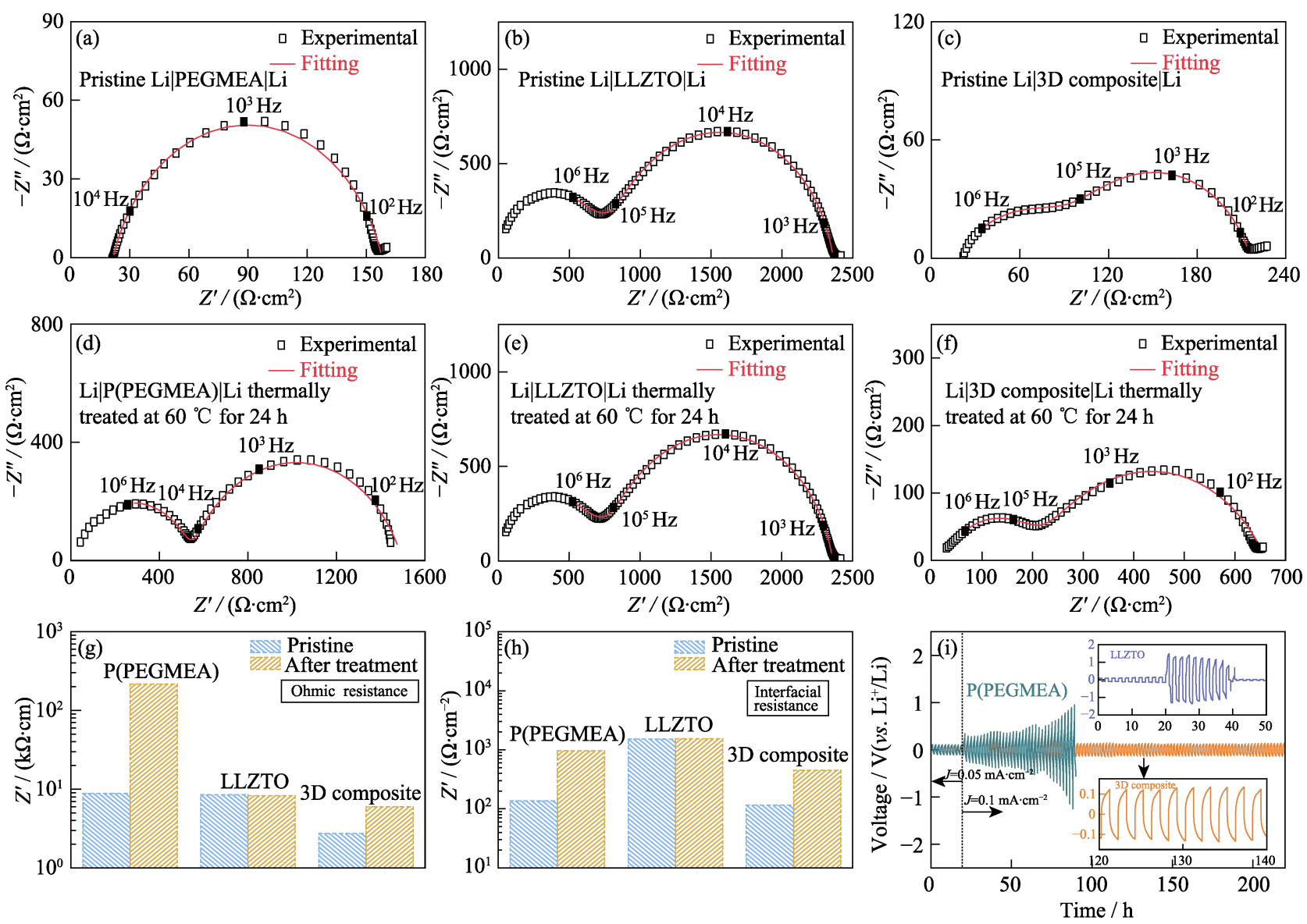

图 4 热处理 $(\mathrm{a} \sim \mathrm{c})$ 前 $(\mathrm{d} \sim \mathrm{f})$ 后基于(a,d)PEGMEA、(b,e)LLZTO 和(c, f)3D composite 的 Li-Li 对称电池的 EIS 图谱; 基于不同电解质的 Li-Li 电池处理前后(g)欧姆阻抗和(h)界面阻抗对比; (i)P(PEGMEA)和 3D composite 的

$\mathrm{Li}-\mathrm{Li}$ 电池室温下的直流恒流循环曲线(上插图为 LLZTO 的 Li-Li 电池室温下的直流恒流循环曲线,

下插图为 $3 \mathrm{D}$ composite 的 $\mathrm{Li}-\mathrm{Li}$ 电池的局部放大极化曲线, 电流密度为 $0.1 \mathrm{~mA} \cdot \mathrm{cm}^{-2}$ )

Fig. 4 EIS plots of (a-c) pre- and (d-f) post-treated Li-Li symmetrical batteries based on (a, d) PEGMEA, (b, e) LLZTO, (c, f) 3D composites; (g) Ohmic and (h) interfacial resistance comparison of pre- and post-treated Li-Li symmetrical cells;

(i) DC galvanostatic cycle of $\mathrm{Li}-\mathrm{Li}$ symmetrical batteries based on P(PEGMEA) and the 3D composite under room temperature at $0.1 \mathrm{~mA} \cdot \mathrm{cm}^{-2}$ with insets showing D.C. galvanostatic cycle of Li-Li symmetrical battery based on LLZTO(up) and the magnified profile of $\mathrm{Li} \mid 3 \mathrm{D}$ composite $\mid \mathrm{Li}($ down) 
加热后, $\mathrm{Li} \mid 3 \mathrm{D}$ composite $\mid \mathrm{Li}$ 的欧姆阻抗增至 $5996 \Omega \cdot \mathrm{cm}$, 而 Li|P(PEGMEA)|Li 的欧姆阻抗陡增至 $216743 \Omega \cdot \mathrm{cm}$ 。 同时, 加热后 $\mathrm{Li} \mid 3 \mathrm{D}$ composite $\mid \mathrm{Li}$ 的界面阻抗从 $115 \Omega \cdot \mathrm{cm}^{2}$ 增加至 $449 \Omega \cdot \mathrm{cm}^{2}$, 不到 $\mathrm{Li}|\mathrm{P}(\mathrm{PEGMEA})| \mathrm{Li}$ 界面阻抗 $956 \Omega \cdot \mathrm{cm}^{2}$ 的二分之一。3D composite 与 P(PEGMEA) 欧姆阻抗和界面阻抗的明显差异, 说 明 p-LLZTO 在提升电导率上的巨大作用。图 4(b,e) 为 $\mathrm{Li}|\mathrm{LLZTO}| \mathrm{Li}$ 热处理前后的 EIS 图谱, 其欧姆阻 抗和界面阻抗几乎没有变化, 分别约为 $8360 \Omega \cdot \mathrm{cm}$ 和 $1540 \Omega \cdot \mathrm{cm}^{2}$, 表明 LLZTO 对锂金属稳定, 无化学 反应发生，与文献[3]报道相符。而 3D composite 的 界面阻抗仅为 LLZTO 的三分之一说明原位聚合策 略能够有效降低界面阻抗。

P(PEGMEA)、LLZTO 和 3D composite 与锂金 属的相容性通过室温下的直流极化进行测试。图 4(i) 中, 电流密度为 $0.1 \mathrm{~mA} \cdot \mathrm{cm}^{-2}$ 时, $\mathrm{Li} \mid 3 \mathrm{D}$ composite $\mid \mathrm{Li}$ 的极化电压仅为 $0.12 \mathrm{~V}$ 且能够稳定循环超过 $200 \mathrm{~h}$, 说明 $3 \mathrm{D}$ composite 与锂金属具有良好的相容性。作 为对比的 $\mathrm{P}(\mathrm{PEGMEA})$ 在 $0.1 \mathrm{~mA} \cdot \mathrm{cm}^{-2}$ 的电流密度下, 不到 $90 \mathrm{~h}$ 其极化电压从 $0.33 \mathrm{~V}$ 增至 $0.95 \mathrm{~V}$ 。这应当归 因于 $\mathrm{P}(\mathrm{PEGMEA})$ 低的室温电导率 $\left(3.6 \times 10^{-6} \mathrm{~S} \cdot \mathrm{cm}^{-1}\right)$ 。
而致密 LLZTO 在电流密度为 $0.1 \mathrm{~mA} \cdot \mathrm{cm}^{-2}$ 时, 其电 池循环不到 $20 \mathrm{~h}$ 就出现短路, 与文献[32]报道相符。 这是由于 LLZTO 与金属锂接触不紧密，局部产生 较大的电流密度, 造成锂的不均匀沉积并使锂枝晶 沿 LLZTO 中的晶界生长, 最终导致电池短路。相较 之下, 利用原位聚合得到的 $3 \mathrm{D}$ composite 与金属锂 之间接触紧密, 电场分布均匀, 因此 3D composite 能够有效抑制锂枝晶的生长 ${ }^{[33-34]}$ 。上述测试结果说 明 $3 \mathrm{D}$ composite 与锂金属有很好的相容性, 能够应 用于 ASLB。

\section{$2.43 \mathrm{D}$ composite 在 ASLB 中的应用}

以 $\mathrm{LiCoO}_{2} \mid \mathrm{Li}$ ASLB 对 $3 \mathrm{D}$ composite 的性能进 行测试。为探究原位聚合策略对 ASLB 的积极作用, 即图 1 中利用原位聚合形成一体化界面, 本工作对 原位聚合及非原位聚合的 $\mathrm{LiCoO}_{2} \mid \mathrm{Li}$ ASLBs 性能进 行对比。 $\mathrm{LiCOO}_{2} \mid \mathrm{Li} \mathrm{ASLB}$ 工作电压范围为 3.0 4.3 V (vs. $\mathrm{Li}^{+} / \mathrm{Li}$ ), 工作温度 $60{ }^{\circ} \mathrm{C}$ 。原位聚合 $\mathrm{LiCoO}_{2} \mid 3 \mathrm{D}$ composite $\mid \mathrm{Li}$ ASLB 在 $0.1 C\left(1 C=140 \mathrm{mAh} \cdot \mathrm{g}^{-1}\right)$ 电流密 度下, 首圈放电比容量为 $144 \mathrm{mAh} \cdot \mathrm{g}^{-1}$, 首圈库仑效 率为 94\%(图 5(a, b))。电流密度为 $0.1 C 、 0.3 C 、 0.5 C$ 时, 其放电比容量分别为 $144 、 138$ 和 $129 \mathrm{mAh} \cdot \mathrm{g}^{-1}$ 。
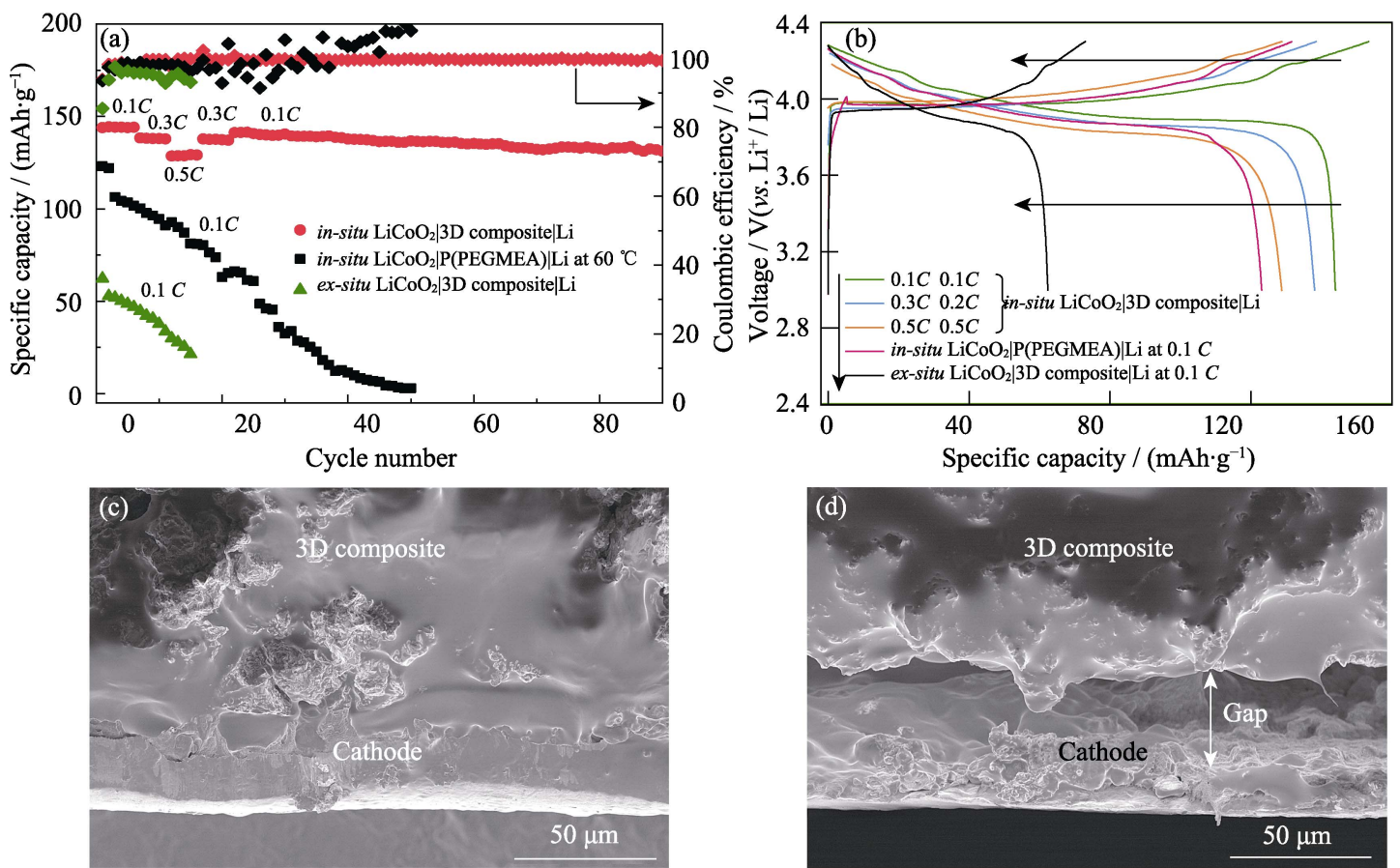

图 5 (a)原位聚合 $\mathrm{LiCoO}_{2} \mid 3 \mathrm{D}$ composite $\mid \mathrm{Li}$ 、原位聚合 $\mathrm{LiCoO}_{2}|\mathrm{P}(\mathrm{PEGMEA})| \mathrm{Li}$ 和非原位聚合 $\mathrm{LiCoO}_{2} \mid 3 \mathrm{D}$ composite $\mid \mathrm{Li}$ ASLBs 的循环性能; (b)原位聚合 $\mathrm{LiCoO}_{2} \mid 3 \mathrm{D}$ composite $\mid \mathrm{Li}$ 、原位聚合 $\mathrm{LiCoO}_{2}|\mathrm{P}(\mathrm{PEGMEA})| \mathrm{Li}$ 和非原位聚合 $\mathrm{LiCoO}{ }_{2} \mid 3 \mathrm{D}$ composite $\mid \mathrm{Li}$ ASLBs 的充放电曲线; (c)原位聚合和(d)非原位聚合 $\mathrm{LiCoO}_{2} \mid 3 \mathrm{D}$ composite $\mid \mathrm{Li}$ ASLBs 拆解后的 $\mathrm{LiCoO}_{2} / 3 \mathrm{D}$ composite 界面的截 面 SEM 照片

Fig. 5 (a) Cycle performances of in-situ $\mathrm{LiCoO}_{2} \mid 3 \mathrm{D}$ composite $\mid \mathrm{Li}$, in-situ $\mathrm{LiCoO}_{2}|\mathrm{P}(\mathrm{PEGMEA})| \mathrm{Li}$, ex-situ LiCoO ${ }_{2} \mid 3 \mathrm{D}$ composite $\mid \mathrm{Li}$ ASLBs; (b) Charge-discharge curves of in-situ $\mathrm{LiCoO}_{2} \mid 3 \mathrm{D}$ composite $\mid \mathrm{Li}$, in-situ $\mathrm{LiCoO}_{2}|\mathrm{P}(\mathrm{PEGMEA})| \mathrm{Li}$, ex-situ LiCoO $2 \mid 3 \mathrm{D}$ composite|Li ASLBs; Cross-sectional SEM images of the $\mathrm{LiCoO}_{2} / 3 \mathrm{D}$ composite interface from the disassembled (c) in-situ and (d) ex-situ $\mathrm{LiCoO}_{2} \mid 3 \mathrm{D}$ composite|Li ASLBs 
在 $0.1 C$ 循环 90 圈后，其容量保持率为 $88 \%$ 。对于 原位聚合 $\mathrm{LiCoO}_{2}|\mathrm{P}(\mathrm{PEGMEA})| \mathrm{Li}$ ASLB, 即使在 $60{ }^{\circ} \mathrm{C}$ 下, 首圈放电比容量也仅为 $123 \mathrm{mAh} \cdot \mathrm{g}^{-1}$, 且 在 40 圈以内迅速衰减至 $10 \mathrm{mAh} \cdot \mathrm{g}^{-1}$ 。而非原位聚 合的 $\mathrm{LiCoO}_{2} \mid 3 \mathrm{D}$ composite $\mid \mathrm{Li}$ ASLB 的性能则更差, 首圈放电比容量仅为 $62 \mathrm{mAh} \cdot \mathrm{g}^{-1}$, 在 15 圈左右便 失效。

分析上述 ASLBs 的失效机理, 首先, 低的离子 电导率是造成原位聚合 $\mathrm{LiCoO}_{2}|\mathrm{P}(\mathrm{PEGMEA})| \mathrm{Li}$ ASLB 性能不佳的主要原因, 即使在 $60{ }^{\circ} \mathrm{C}$ 下, P(PEGMEA) 的电导率也仅为 $1.85 \times 10^{-5} \mathrm{~S} \cdot \mathrm{cm}^{-1}$, 这严重限制了 $\mathrm{Li}^{+}$ 的快速输运。对于非原位聚合 $\mathrm{LiCoO}_{2} \mid 3 \mathrm{D}$ composite $\mid \mathrm{Li}$ ASLB, 虽然 3D composite 具有较高的电导率, 但电极与电解质之间存在约 $30 \mu \mathrm{m}$ 的间隙, 不连续 的界面接触严重阻碍了 $\mathrm{Li}^{+}$在电解质-电极之间的传 输, 造成电池性能的快速衰减。如图 5(c, d) 所示, 通 过原位聚合制备的 $\mathrm{LiCoO}_{2} \mid 3 \mathrm{D}$ composite $\mid \mathrm{Li}$ 具有一 体化的电解质/电极界面, 确保 $\mathrm{Li}^{+}$在界面处顺利地 传输。上述三种 ASLBs 的性能对比证明了高的电导 率和一体化的电解质-电极界面是获得高性能 ASLB 的必要条件, 而本工作中通过原位聚合的策略成功 实现了高电导率 3D composite 与电极之间的一体化 界面的构建。

\section{3 结论}

综上, 本工作以石墨粉为造孔剂通过高温烧结 成功制备自支撑三维多孔 $\mathrm{Li}_{6.4} \mathrm{Al}_{0.1} \mathrm{La}_{3} \mathrm{Zr}_{1.7} \mathrm{Ta}_{0.3} \mathrm{O}_{12}$ 骨 架。将聚乙二醇甲基醚丙烯酸酯浇注于多孔 $\mathrm{Li}_{6.4} \mathrm{Al}_{0.1} \mathrm{La}_{3} \mathrm{Zr}_{1.7} \mathrm{Ta}_{0.3} \mathrm{O}_{12}$ 中, 聚合后得到三维有机无 机复合电解质。连续的 $\mathrm{Li}_{6.4} \mathrm{Al}_{0.1} \mathrm{La}_{3} \mathrm{Zr}_{1.7} \mathrm{Ta}_{0.3} \mathrm{O}_{12}$ 相能 够为 $\mathrm{Li}^{+}$的快速传输提供通道, 并将聚合后的聚乙二 醇甲基醚丙烯酸酯的室温电导率提升 53 倍，达到 $1.9 \times 10^{-4} \mathrm{~S} \cdot \mathrm{cm}^{-1}$ 。更重要的是, 原位聚合的聚乙二醇 甲基醚丙烯酸酯能够在接触不良的三维有机无机复 合电解质和电极之间形成一体化界面, 有效地将电 池界面阻抗从 $1542 \Omega \cdot \mathrm{cm}^{2}$ 降低至 $449 \Omega \cdot \mathrm{cm}^{2}$ 。最后, 原位聚合三维有机无机复合电解质被成功应用于 $\mathrm{LiCoO}_{2} \mid \mathrm{Li}$ 全固态锂电池。本工作为制备与高电压正 极、锂负极有良好化学机械相容性的高电导率有机/ 无机复合电解质提供了有价值的参考。

\section{补充材料}

本文相关补充材料可登陆 https://doi.org/ 10.15541/ jim20200152 查看。

\section{参考文献:}

[1] GAO Z, SUN H, FU L, et al. Promises, challenges, and recent progress of inorganic solid-state electrolytes for all-solid-state lithium batteries. Advanced Materials, 2018, 30(17): e1705702.

[2] BACHMAN J C, MUY S, GRIMAUD A, et al. Inorganic solid-state electrolytes for lithium batteries: mechanisms and properties governing ion conduction. Chemical Reviews, 2016, 116(1): $140-162$.

[3] ZHENG F, KOTOBUKI M, SONG S, et al. Review on solid electrolytes for all-solid-state lithium-ion batteries. Journal of Power Sources, 2018, 389: 198-213.

[4] ZHANG B, TAN R, YANG L, et al. Mechanisms and properties of ion-transport in inorganic solid electrolytes. Energy Storage Materials, 2018, 10: 139-159.

[5] CHEN R, QU W, GUO X, et al. The pursuit of solid-state electrolytes for lithium batteries: from comprehensive insight to emerging horizons. Materials Horizons, 2016, 3(6): 487-516.

[6] FAN L, WEI S, LI S, et al. Recent progress of the solid-state electrolytes for high-energy metal-based batteries. Advanced Energy Materials, 2018, 8 (11): 1702657.

[7] YUE L, MA J, ZHANG J, et al. All solid-state polymer electrolytes for high-performance lithium ion batteries. Energy Storage Materials, 2016, 5: 139-164.

[8] MANTHIRAM A, YU X, WANG S. Lithium battery chemistries enabled by solid-state electrolytes. Nature Reviews Materials, 2017, 2(4): 16103

[9] GAO Y, WANG D, LI Y C, et al. Salt-based organic-inorganic nanocomposites: towards a stable lithium metal $/ \mathrm{Li}_{10} \mathrm{GeP}_{2} \mathrm{~S}_{12}$ solid electrolyte interface. Angew. Chem. Int. Ed., 2018, 57(41): 13608-13612.

[10] BUANNIC L, ORAYECH B. Dual substitution strategy to enhance $\mathrm{Li}^{+}$ionic conductivity in $\mathrm{Li}_{7} \mathrm{La}_{3} \mathrm{Zr}_{2} \mathrm{O}_{12}$ solid electrolyte. Chemistry of Materials, 2017, 29(4): 1769-1778.

[11] ZHANG Z, SHAO Y, LOTSCH B, et al. New horizons for inorganic solid state ion conductors. Energy \& Environmental Science, 2018, 11(8): 1945-1976.

[12] CHENG X B, ZHAO C Z, YAO Y X, et al. Recent advances in energy chemistry between solid-state electrolyte and safe lithiummetal anodes. Chem, 2019, 5(1): 74-96.

[13] ZHA W, CHEN F, YANG D, et al. High-performance $\mathrm{Li}_{6.4} \mathrm{La}_{3} \mathrm{Zr}_{1.4} \mathrm{Ta}_{0.6} \mathrm{O}_{12} /$ poly(ethylene oxide)/succinonitrile composite electrolyte for solid-state lithium batteries. Journal of Power Sources, 2018, 397: 87-94.

[14] ZHU P, YAN C, DIRICAN M, et al. $\mathrm{Li}_{0.33} \mathrm{La}_{0.557} \mathrm{TiO}_{3}$ ceramic nanofiberenhanced polyethylene oxide-based composite polymer electrolytes for all-solid-state lithium batteries. Journal of Materials Chemistry A, 2018, 6(10): 4279-4285.

[15] WAN Z, LEI D, YANG $\mathrm{W}$, et al. Low resistance-integrated all-solid-state battery achieved by $\mathrm{Li}_{7} \mathrm{La}_{3} \mathrm{Zr}_{2} \mathrm{O}_{12}$ nanowire upgrading polyethylene oxide (PEO) composite electrolyte and PEO cathode binder. Advanced Functional Materials, 2019, 29(1): 1805301.

[16] CHEN L, LI Y, LI S P, et al. PEO/garnet composite electrolytes for solid-state lithium batteries: from "ceramic-in-polymer" to "polymerin-ceramic". Nano Energy, 2018, 46: 176-184.

[17] XIE H, YANG C, FU K K, et al. Flexible, scalable, and highly conductive garnet-polymer solid electrolyte templated by bacterial cellulose. Advanced Energy Materials, 2018, 8(18): 1703474.

[18] BAE J, LI Y, ZHANG J, et al. A 3D nanostructured hydrogelframework-derived high-performance composite polymer lithium-ion electrolyte. Angew. Chem. Int. Ed., 2018, 57(8): 2096-2100.

[19] BAE J, LI Y, ZHAO F, et al. Designing 3D nanostructured garnet 
frameworks for enhancing ionic conductivity and flexibility in composite polymer electrolytes for lithium batteries. Energy Storage Materials, 2018, 15: 46-52.

[20] LIU Y, SUN Q, ZHAO Y, et al. Stabilizing the interface of NASICON solid electrolyte against Li metal with atomic layer deposition. ACS Applied Materials \& Interfaces, 2018, 10(37): 31240-31248.

[21] JU J, WANG Y, CHEN B, et al. Integrated interface strategy toward room temperature solid-state lithium batteries. ACS Applied Materials \& Interfaces, 2018, 10(16): 13588-13597.

[22] ZHAO Q, LIU X, STALIN S, et al. Solid-state polymer electrolytes with in-built fast interfacial transport for secondary lithium batteries. Nature Energy, 2019, 4(5): 365-373.

[23] DUAN H, YIN Y X, SHI Y, et al. Dendrite-free Li-metal battery enabled by a thin asymmetric solid electrolyte with engineered layers. Journal of the American Chemical Society, 2018, 140(1): 82-85.

[24] PARANJAPE N, MANDADAPU P C, WU G, et al. Highlybranched cross-linked poly(ethylene oxide) with enhanced ionic conductivity. Polymer, 2017, 111: 1-8.

[25] BAN X, ZHANG W, CHEN N, et al. A high-performance and durable poly(ethylene oxide)-based composite solid electrolyte for all solid-state lithium battery. The Journal of Physical Chemistry C, 2018, 122(18): 9852-9858.

[26] GONG Y, FU K, XU S, et al. Lithium-ion conductive ceramic textile: a new architecture for flexible solid-state lithium metal batteries. Materials Today, 2018, 21(6): 594-601.
[27] LI D, CHEN L, WANG T, et al. 3D fiber-network-reinforced bicontinuous composite solid electrolyte for dendrite-free lithium metal batteries. ACS Applied Materials \& Interfaces, 2018, 10(8): 7069-7078.

[28] LI Z, HUANG H M, ZHU J K, et al. Ionic conduction in composite polymer electrolytes: case of PEO:Ga-LLZO composites. ACS Applied Materials \& Interfaces, 2019, 11(1): 784-791.

[29] WANG Q, WEN Z, JIN J, et al. A gel-ceramic multi-layer electrolyte for long-life lithium sulfur batteries. Chem. Commun. (Camb), 2016, 52(8): 1637-1640.

[30] HAN X, GONG Y, FU K K, et al. Negating interfacial impedance in garnet-based solid-state Li metal batteries. Nature Materials, 2017, 16(5): 572-579.

[31] JU J, CHEN F, XIA C. Ionic conductivity of impregnated samaria doped ceria for solid oxide fuel cells. Electrochimica Acta, 2014, 136: $422-429$.

[32] WU B, WANG S, LOCHALA J, et al. The role of the solid electrolyte interphase layer in preventing Li dendrite growth in solid-state batteries. Energy \& Environmental Science, 2018, 11(7): 1803-1810.

[33] HU J L, TIAN J, Li C L. Nanostructured carbon nitride polymerreinforced electrolyte to enable dendrite-suppressed lithium metal batteries. ACS Applied Materials \& Interfaces, 2017, 9: 11615-11625.

[34] HU J L, YAO Z G, CHEN K Y, et al. High-conductivity open framework fluorinated electrolyte bonded by solidified ionic liquid wires for solid-state Li metal batteries. Energy Storage Materials, 2020, 28: 37-46. 